\title{
Flavor-changing hyperon decays with light invisible bosons
}

\author{
Gang $\mathrm{Li},{ }^{1}$ Jhih-Ying Su, ${ }^{1}$ and Jusak Tandean $\circledast^{1,2}$ \\ ${ }^{1}$ Department of Physics, National Taiwan University, Taipei 106, Taiwan \\ ${ }^{2}$ Physics Division, National Center for Theoretical Sciences, Hsinchu 300, Taiwan
}

(Received 31 May 2019; published 2 October 2019)

\begin{abstract}
We consider the strangeness-changing decays of hyperons into another baryon and missing energy which is carried away by a pair of invisible spinless bosons. Pursuing a model-independent approach and taking into account constraints from the kaon sector, we find that these hyperon modes can have large rates, especially if the bosons have parity-odd effective couplings to light quarks. In that case the rates could attain values which are well within the expected reach of the currently running BESIII experiment. Since the branching fractions of hyperon decays with missing energy are tiny in the standard model, observing them at ongoing or upcoming facilities would likely be indicative of new physics. In addition, we show that the kaon restrictions may be weakened or absent if the bosons have nonnegligible mass. Specifically, if the bosons interact with the quarks solely via an axial-vector current, in the mass region from about 115 to $175 \mathrm{MeV}$ the constraints from rare kaon decays no longer apply and a couple of the hyperon modes become the only direct probes of such interactions. We also make comparisons with the previously explored newphysics scenario where the invisible particles are spin- $1 / 2$ fermions.
\end{abstract}

DOI: 10.1103/PhysRevD.100.075003

\section{INTRODUCTION}

In the standard model (SM) the flavor-changing neutral current (FCNC) decay of a strange meson or baryon with missing energy $(\mathbb{E})$ in the final state proceeds mainly from the short-distance contribution due to the quark transition $s \rightarrow d \nu \bar{\nu}$ generated by loop diagrams [1], with $\not E$ being carried away by the unobserved neutrino pair $(\nu \bar{\nu})$. Beyond the SM there could be extra ingredients which cause changes to the SM process and/or give rise to additional channels with one or more invisible nonstandard particles adding to $\boldsymbol{E}$. As such decay modes of strange hadrons have very suppressed rates in the SM, the modifications due to new physics (NP) may translate into effects big enough to be discoverable. These rare modes can then be expected to serve as sensitive probes of NP.

Experimentally, there has been much activity to study such processes in the meson sector. Particularly, a while ago the E949 Collaboration [2] reported seeing the kaon mode $K^{+} \rightarrow \pi^{+} \nu \bar{\nu}$, and currently there are running programs to measure it more precisely and observe the neutral channel $K_{L} \rightarrow \pi^{0} \nu \bar{\nu}$ by the NA62 [3] and KOTO [4] Collaborations, respectively. As for other FCNC kaon decays with missing energy, the only data available are upper limits on the

Published by the American Physical Society under the terms of the Creative Commons Attribution 4.0 International license. Further distribution of this work must maintain attribution to the author(s) and the published article's title, journal citation, and DOI. Funded by SCOAP ${ }^{3}$. branching fractions of two of the $K \rightarrow \pi \pi^{\prime} \nu \bar{\nu}$ modes from quests for them with negative outcomes [5-7]. Indirectly, the corresponding limits on $K \rightarrow \not \mathbb{E}$ can be inferred from the existing data on the neutral kaon visible decays [8].

In the baryon sector, the analogous transitions are the strangeness-changing $(|\Delta S|=1)$ hyperon decays into another baryon plus missing energy. However, there has never been any search for them as far as we know. Interestingly, there is a recent proposal to measure them in the BESIII experiment [9]. Hence new data on these rare hyperon modes may be forthcoming.

It is therefore timely to investigate them theoretically to learn whether NP might impact them significantly in light of the constraints from the kaon sector. Initial works on the hyperon modes have been done in Refs. $[9,10]$ focusing on NP entering through $s \rightarrow d \nu \bar{\nu}$ interactions with the same chiral structure as in the SM. Subsequently, Ref. [11] explores a more general scenario in which the underlying NP operators involve other Lorentz structures and the pair of invisible particles could be nonstandard spin- $1 / 2$ fermions. In this more general possibility, it turns out that the hyperon decays could have rates which are greatly increased relative to the SM expectations, even up to levels potentially detectible by BESIII in the near future [11].

In the present paper, we extend the analysis of Ref. [11] and entertain the possibility that the invisible pair consists of a spin- 0 boson, $\phi$, and its antiparticle, $\bar{\phi}$, rather than fermions. We assume that the $\phi$ field is complex and does not carry any SM gauge charge but is either charged under some symmetry of a dark sector beyond the SM or odd 
under a $Z_{2}$ symmetry which does not influence SM fields. Moreover, we suppose that any other states beyond the SM are heavier than the weak scale. As a consequence, $\phi$ and $\bar{\phi}$ always appear together in their interactions with SM quarks at low energies. Scenarios where such nonstandard bosons have $d s$ couplings that contribute to kaon processes have been addressed before in the literature under specific NP contexts [12-15]. Here we pursue a model-independent approach, starting from the most general effective Lagrangian that satisfies SM gauge invariance and contains operators describing $d s \phi \phi$ interactions at leading order.

We will show that the rates of $|\Delta S|=1$ hyperon decays with missing energy can be sizable in the presence of the new interactions, similarly to what happens in the invisible fermion case studied in Ref. [11]. Such a possibility is partly attributable to the fact that these hyperon transitions and their kaon counterparts do not probe the same set of the underlying NP operators. In particular, $K \rightarrow \pi E$ is sensitive exclusively to parity-even $d s \phi \phi$ operators and $K \rightarrow \mathbb{E}$ to the parity-odd ones, while $K \rightarrow \pi \pi^{\prime} \mathbb{E}$ and the spin-1/2 hyperon modes can probe both. Another factor is the kaon data situation at the moment, which has a bearing on limiting mainly the parity-even operators, due to the existing empirical information on $K \rightarrow \pi \nu \bar{\nu}$ decays [3-5]. By contrast, the restraints from $K \rightarrow \mathbb{E}$ and $K \rightarrow \pi \pi^{\prime} \mathbb{E}$ are relatively far weaker and still permit the parity-odd operators to bring about considerable amplifying effects on the hyperon rates.

Besides the similarities, there are differences between the bosonic and fermionic cases. Due to the invisible particle having spin $1 / 2$, the latter scenario involves more operators with their associated coupling parameters, leading to more possibilities in the phenomenology, especially if the invisible particle's mass is not negligible. Our examples will illustrate the salient differences and similarities.

The structure of the remainder of the paper is as follows. In the next section we write down the $d s \phi \phi$ operators of interest, treating them in a model-independent manner. In Sec. III we first deal with the FCNC hyperon decays with missing energy, deriving their differential rates, and subsequently provide the formulas for the corresponding kaon decays of concern. In Sec. IV we present our numerical results. Evaluating the hyperon modes and taking into account restrictions from the kaon sector, we demonstrate that the hyperon rates can be substantial and within the proposed BESIII reach. We also make comparisons with the fermionic case examined in Ref. [11], where in the numerical analysis the invisible fermion's mass was assumed to be negligible or zero for simplicity, and furthermore discuss what may happen if it is not negligible. In the latter case, we point out that under certain conditions a few of the hyperon modes are allowed to have rates higher than their counterparts computed in Ref. [11]. In Sec. V we give our conclusions.

\section{INTERACTIONS}

At lowest order, the effective operators responsible for the SM-gauge-invariant interactions of $\phi$ with quarks as specified above are of mass dimension six [16,17]. The relevant Lagrangian $\mathcal{L}_{\mathrm{NP}}$ is given by [17]

$$
\begin{aligned}
-\Lambda_{\mathrm{NP}}^{2} \mathcal{L}_{\mathrm{NP}}= & \left(\mathcal{C}_{j k} \bar{q}_{j} \gamma^{\eta} q_{k}+\mathcal{C}_{j k}^{\prime} \bar{d}_{j} \gamma^{\eta} d_{k}\right) i\left(\phi^{\dagger} \partial_{\eta} \phi\right. \\
& \left.-\partial_{\eta} \phi^{\dagger} \phi\right)+\left(\mathcal{C}_{j k}^{\prime \prime} \bar{q}_{j} d_{k} H+\text { H.c. }\right) \phi^{\dagger} \phi,
\end{aligned}
$$

where $\Lambda_{\mathrm{NP}}$ is a heavy mass scale representing the underlying NP interactions, $\mathcal{C}_{j k}^{(, \prime \prime)}$ in our model-independent approach are dimensionless free parameters which are generally complex, $q_{j}$ and $d_{j}$ denote a left-handed quark doublet and right-handed down-type quark singlet, respectively, $H$ stands for the Higgs doublet, and summation over family indices $j, k=1,2,3$ is implicit. ${ }^{1}$ The Hermiticity of $\mathcal{L}_{\mathrm{NP}}$ implies $\mathcal{C}_{j k}^{(\prime)}=\mathcal{C}_{k j}^{(\prime) *}$.

To deal with the contributions of $\mathcal{L}_{\mathrm{NP}}$ to the hyperon and kaon decays, it is convenient to rewrite the $d s \phi \phi$ operators in it explicitly separating their parity-even and -odd parts. Thus, choosing to work in the mass basis of the down-type quarks, at low energies we can express

$$
\begin{aligned}
-\mathcal{L}_{\mathrm{NP}} \supset & \left(\mathrm{c}_{\phi}^{\mathrm{V}} \bar{d} \gamma^{\eta} s+\mathrm{c}_{\phi}^{\mathrm{A}} \bar{d} \gamma^{\eta} \gamma_{5} s\right) i\left(\phi^{\dagger} \partial_{\eta} \phi-\partial_{\eta} \phi^{\dagger} \phi\right) \\
& +\left(\mathrm{c}_{\phi}^{\mathrm{S}} \bar{d} s+\mathrm{c}_{\phi}^{\mathrm{P}} \bar{d} \gamma_{5} s\right) \phi^{\dagger} \phi+\text { H.c. },
\end{aligned}
$$

where

$$
\begin{array}{ll}
\mathrm{c}_{\phi}^{\mathrm{V}}=\frac{\mathcal{C}_{12}+\mathcal{C}_{12}^{\prime}}{2 \Lambda_{\mathrm{NP}}^{2}}, & \mathrm{c}_{\phi}^{\mathrm{A}}=\frac{-\mathcal{C}_{12}+\mathcal{C}_{12}^{\prime}}{2 \Lambda_{\mathrm{NP}}^{2}}, \\
\mathrm{c}_{\phi}^{\mathrm{S}}=\frac{\mathcal{C}_{12}^{\prime \prime}+\mathcal{C}_{21}^{\prime \prime *}}{2 \sqrt{2} \Lambda_{\mathrm{NP}}^{2}} v, & \mathrm{c}_{\phi}^{\mathrm{P}}=\frac{\mathcal{C}_{12}^{\prime \prime}-\mathcal{C}_{21}^{\prime \prime *}}{2 \sqrt{2} \Lambda_{\mathrm{NP}}^{2}} v,
\end{array}
$$

with $v \simeq 246 \mathrm{GeV}$ being the Higgs vacuum expectation value. $^{2}$ As we will see below, in the decay rates the contributions of $\mathrm{c}_{\phi}^{\mathrm{V}, \mathrm{A}, \mathrm{S}, \mathrm{P}}$ do not interfere. In numerical calculations, we will adopt the phenomenological viewpoint that these free parameters can have any values compatible with the empirical constraints and perturbativity, implying that some of them may be taken to be vanishing or much less than the others.

\section{AMPLITUDES AND RATES}

\section{A. Baryon decays}

Our hyperon decays of interest are $\mathfrak{B} \rightarrow \mathfrak{B}^{\prime} \phi \bar{\phi}$ with $\mathfrak{B} \mathfrak{B}^{\prime}=\Lambda n, \Sigma^{+} p, \Xi^{0} \Lambda, \Xi^{0} \Sigma^{0}, \Xi^{-} \Sigma^{-}$, all of the baryons

\footnotetext{
${ }^{1}$ If the $\phi$ field is real instead, the $\mathcal{C}_{j k}$ and $\mathcal{C}_{j k}^{\prime}$ terms are absent from Eq. (1).

${ }^{2} \mathrm{We}$ mention that for effective flavor-changing operators involving light quarks and SM-gauge-singlet dark particles the running of their coefficients from high to low energies has been estimated to be negligible [18].
} 
having spin $1 / 2$, and $\Omega^{-} \rightarrow \Xi^{-} \phi \bar{\phi}$ where $\Omega^{-}$has spin $3 / 2$. One or more of these modes may be searched for at BESIII which can produce copious $\Lambda, \Sigma, \Xi$, and $\Omega$ hyperons [9].

To derive the amplitudes for these decays, we need the baryonic matrix elements of the quark portions of the operators in Eq. (2). They can be estimated with the aid of flavor-SU(3) chiral perturbation theory at leading order $[11,19]$. For $\mathfrak{B} \rightarrow \mathfrak{B}^{\prime} \phi \bar{\phi}$, from Ref. [11] we have

$$
\begin{aligned}
\left\langle\mathfrak{B}^{\prime}\left|\bar{d} \gamma^{\eta} s\right| \mathfrak{B}\right\rangle & =\mathcal{V}_{\mathfrak{B}^{\prime} \mathfrak{B}} \bar{u}_{\mathfrak{B}^{\prime} \gamma^{\eta} u_{\mathfrak{B}},} \\
\left\langle\mathfrak{B}^{\prime}\left|\bar{d} \gamma^{\eta} \gamma_{5} s\right| \mathfrak{B}\right\rangle & =\bar{u}_{\mathfrak{B}^{\prime}}\left(\gamma^{\eta} \mathcal{A}_{\mathfrak{B}^{\prime} \mathfrak{B}}-\frac{\mathcal{P}_{\mathfrak{B}^{\prime} \mathfrak{B}}}{B_{0}} Q^{\eta}\right) \gamma_{5} u_{\mathfrak{B}}, \\
\left\langle\mathfrak{B}^{\prime}|\bar{d} s| \mathfrak{B}\right\rangle & =\mathcal{S}_{\mathfrak{B}^{\prime} \mathfrak{B}} \bar{u}_{\mathfrak{B}^{\prime}} u_{\mathfrak{B}}, \\
\left\langle\mathfrak{B}^{\prime}\left|\bar{d} \gamma_{5} s\right| \mathfrak{B}\right\rangle & =\mathcal{P}_{\mathfrak{B}^{\prime} \mathfrak{B}} \bar{u}_{\mathfrak{B}^{\prime}} \gamma_{5} u_{\mathfrak{B}},
\end{aligned}
$$

where $\mathcal{V}_{\mathfrak{B}^{\prime} \mathfrak{B}}=-3 / \sqrt{6},-1,3 / \sqrt{6},-1 / \sqrt{2}, 1$ and $\mathcal{A}_{\mathfrak{B}^{\prime} \mathfrak{B}}=$ $-(D+3 F) / \sqrt{6}, D-F,(3 F-D) / \sqrt{6}, \quad-(D+F) / \sqrt{2}$, $D+F$ for $\mathfrak{B B}^{\prime}=\Lambda n, \Sigma^{+} p, \Xi^{0} \Lambda, \Xi^{0} \Sigma^{0}, \Xi^{-} \Sigma^{-}$, respectively, $\bar{u}_{\mathfrak{B}^{\prime}}$ and $u_{\mathfrak{B}}$ denote the Dirac spinors of the baryons, $\mathrm{Q}=p_{\mathfrak{B}}-p_{\mathfrak{B}^{\prime}}$, with $p_{\mathfrak{B}}$ and $p_{\mathfrak{B}^{\prime}}$ representing their fourmomenta, $\mathcal{S}_{\mathfrak{B}^{\prime} \mathfrak{B}}=\mathcal{V}_{\mathfrak{B}^{\prime} \mathfrak{B}}\left(m_{\mathfrak{B}}-m_{\mathfrak{B}^{\prime}}\right) /\left(m_{s}-\hat{m}\right)$, and $\mathcal{P}_{\mathfrak{B}^{\prime} \mathfrak{B}}=$ $\mathcal{A}_{\mathfrak{B}^{\prime} \mathfrak{B}} B_{0}\left(m_{\mathfrak{B}^{\prime}}+m_{\mathfrak{B}}\right) /\left(m_{K}^{2}-Q^{2}\right)$, with $m_{\left.\mathfrak{B}^{\prime \prime}\right)}$ and $m_{K}$ in $\mathcal{S}_{\mathfrak{B}^{\prime} \mathfrak{B}}$ and $\mathcal{P}_{\mathcal{B}^{\prime} \mathfrak{B}}$ referring to the isospin-averaged masses of the baryon $\mathfrak{B}^{(\prime)}$ and kaon, $\hat{m}=\left(m_{u}+m_{d}\right) / 2$ and $m_{s}$ being light quarks' masses, and $B_{0}=m_{K}^{2} /\left(\hat{m}+m_{s}\right)$. For
$\Omega^{-} \rightarrow \Xi^{-} \phi \bar{\phi}$, the hadronic matrix elements that do not vanish at lowest order in chiral perturbation theory are [11]

$$
\begin{aligned}
\left\langle\Xi^{-}\left|\bar{d} \gamma^{\eta} \gamma_{5} s\right| \Omega^{-}\right\rangle & =\mathcal{C} \bar{u}_{\Xi}\left(u_{\Omega}^{\eta}+\frac{\tilde{Q}^{\eta} \tilde{Q}_{\kappa}}{m_{K}^{2}-\tilde{Q}^{2}} u_{\Omega}^{\kappa}\right), \\
\left\langle\Xi^{-}\left|\bar{d} \gamma_{5} s\right| \Omega^{-}\right\rangle & =\frac{B_{0} \mathcal{C} \tilde{Q}_{\kappa}}{\tilde{Q}^{2}-m_{K}^{2}} \bar{u}_{\Xi} u_{\Omega}^{\kappa},
\end{aligned}
$$

where $\tilde{Q}=p_{\Omega^{-}}-p_{\Xi^{-}}$and $u_{\Omega}^{\eta}$ is a Rarita-Schwinger spinor. The constants $D, F$, and $\mathcal{C}$ above come from the lowest-order chiral Lagrangian and can be fixed from baryon decay data. In numerical analysis, we will employ the same (central) values of these and other input parameters as those given in Ref. [11].

With Eqs. (4) and (5), we derive the amplitudes for $\mathfrak{B} \rightarrow$ $\mathfrak{B}^{\prime} \phi \bar{\phi}$ and $\Omega^{-} \rightarrow \Xi^{-} \phi \bar{\phi}$ to be

$$
\begin{aligned}
\mathcal{M}_{\mathfrak{B} \rightarrow \mathfrak{B}^{\prime} \phi \bar{\phi}}= & \bar{u}_{\mathcal{B}^{\prime}}\left[\gamma^{\eta}(\mathrm{p}-\overline{\mathrm{p}})_{\eta}\left(\mathrm{c}_{\phi}^{\mathrm{V}} \mathcal{V}_{\mathcal{B}^{\prime} \mathfrak{B}}+\gamma_{5} \mathrm{c}_{\phi}^{\mathrm{A}} \mathcal{A}_{\mathfrak{B}^{\prime} \mathfrak{B}}\right)\right. \\
& \left.+\mathrm{c}_{\phi}^{\mathrm{S}} \mathcal{S}_{\mathfrak{B}^{\prime} \mathfrak{B}}+\gamma_{5} \mathrm{c}_{\phi}^{\mathrm{P}} \mathcal{P}_{\mathfrak{B}^{\prime} \mathfrak{B}}\right] u_{\mathfrak{B}}, \\
\mathcal{M}_{\Omega \rightarrow \Xi \phi \bar{\phi}}= & {\left[\mathrm{c}_{\phi}^{\mathrm{A}}(\mathrm{p}-\overline{\mathrm{p}})_{\eta}+\frac{B_{0} \mathrm{c}_{\phi}^{\mathrm{P}}(\mathrm{p}+\overline{\mathrm{p}})_{\eta}}{(\mathrm{p}+\overline{\mathrm{p}})^{2}-m_{K}^{2}}\right] \mathcal{C} \bar{u}_{\Xi} u_{\Omega}^{\eta}, }
\end{aligned}
$$

where $\mathrm{p}$ and $\overline{\mathrm{p}}$ denote the momenta of $\phi$ and $\bar{\phi}$, respectively. Subsequently, we arrive at the differential decay rates

$$
\begin{aligned}
& \frac{d \Gamma_{\mathfrak{B} \rightarrow \mathfrak{B}^{\prime} \phi \bar{\phi}}}{d \hat{s}}=\frac{\beta \lambda_{\mathfrak{B B}^{\prime}}^{1 / 2}}{256 \pi^{3} m_{\mathfrak{B}^{3}}^{3}}\left[\beta^{2}\left(\frac{\lambda_{\mathfrak{B B}^{\prime}}}{3}+\tilde{\sigma}_{\mathfrak{B B}^{\prime}}^{-} \hat{s}\right)\left|\mathbf{C}_{\phi}^{\mathrm{V}}\right|^{2} \mathcal{V}_{\mathfrak{B}^{\prime} \mathfrak{B}}^{2}+\beta^{2}\left(\frac{\lambda_{\mathfrak{B B}^{\prime}}}{3}+\tilde{\sigma}_{\mathfrak{B B}^{\prime}}^{+} \hat{s}\right)\left|\mathbf{c}_{\phi}^{\mathrm{A}}\right|^{2} \mathcal{A}_{\mathfrak{B}^{\prime} \mathfrak{B}}^{2}\right. \\
& \left.+\tilde{\sigma}_{\mathfrak{B}^{\prime}}^{+}\left|\mathrm{c}_{\phi}^{\mathrm{S}}\right|^{2} \mathcal{S}_{\mathfrak{B}^{\prime} \mathfrak{B}}^{2}+\tilde{\sigma}_{\mathfrak{B B}^{\prime}}^{-}\left|\mathbf{C}_{\phi}^{\mathrm{P}}\right|^{2} \mathcal{P}_{\mathfrak{B}^{\prime} \mathfrak{B}}^{2}\right], \\
& \frac{d \Gamma_{\Omega \rightarrow \Xi \phi \bar{\phi}}}{d \hat{s}}=\frac{\beta \lambda_{\Omega^{-} \Xi^{-}}^{1 / 2} \tilde{\sigma}_{\Omega^{-} \Xi^{-}}^{+} \mathcal{C}^{2}}{3072 \pi^{3} m_{\Omega^{-}}^{5}}\left[\beta^{2}\left(\frac{\lambda_{\Omega^{-} \Xi^{-}}}{3}+4 m_{\Omega^{-} \hat{s}}^{2}\right)\left|\mathrm{C}_{\phi}^{\mathrm{A}}\right|^{2}+\frac{\lambda_{\Omega^{-} \Xi^{-}} B_{0}^{2}}{\left(m_{K}^{2}-\hat{s}\right)^{2}}\left|\mathrm{C}_{\phi}^{\mathrm{P}}\right|^{2}\right],
\end{aligned}
$$

where

$$
\begin{aligned}
& \beta=\sqrt{1-\frac{4 m_{\phi}^{2}}{\hat{s}},} \quad \lambda_{X Y}=\mathcal{K}\left(m_{X}^{2}, m_{Y}^{2}, \hat{s}\right), \quad \mathcal{K}(x, y, z)=(x-y-z)^{2}-4 y z, \\
& \hat{s}=(\mathrm{p}+\overline{\mathrm{p}})^{2}, \quad \tilde{\sigma}_{X Y}^{ \pm}=\left(m_{X} \pm m_{Y}\right)^{2}-\hat{s} .
\end{aligned}
$$

To get the decay rates, we integrate the differential rates in Eq. (7) over $4 m_{\phi}^{2} \leq \hat{s} \leq\left(m_{\mathfrak{B}}-m_{\mathfrak{B}^{\prime}}\right)^{2}$ and $4 m_{\phi}^{2} \leq \hat{s} \leq$ $\left(m_{\Omega^{-}}-m_{\Xi^{-}}\right)^{2}$, respectively.

Following Ref. [11], in our numerical treatment of these hyperon decay rates we will incorporate form-factor effects not yet taken into account in Eqs. (4) and (5). Specifically, we will make the changes $\mathcal{V}_{\mathfrak{B}^{\prime} \mathfrak{B}} \rightarrow\left(1+2 Q^{2} / M_{V}^{2}\right) \mathcal{V}_{\mathfrak{B}^{\prime} \mathfrak{B}}$ and $\mathcal{A}_{\mathfrak{B}^{\prime} \mathfrak{B}} \rightarrow\left(1+2 \mathrm{Q}^{2} / M_{A}^{2}\right) \mathcal{A}_{\mathfrak{B}^{\prime} \mathfrak{B}}$ with $M_{V}=0.97(4) \mathrm{GeV}$ and $M_{A}=1.25(15) \mathrm{GeV}$, which are in line with the commonly used parametrization in experimental studies of hyperon semileptonic decays [20-24]. Moreover, for the $\Omega^{-}$decay we apply the modification $\mathcal{C} \rightarrow \mathcal{C} /\left(1-\tilde{Q}^{2} / M_{A}^{2}\right)^{2}$.

\section{B. Kaon decays}

For $K \rightarrow \phi \bar{\phi}$ and $K \rightarrow \pi \phi \bar{\phi}$, the mesonic matrix elements that do not vanish are given by 


$$
\begin{aligned}
\left\langle 0\left|\bar{d} \gamma^{\eta} \gamma_{5} s\right| \bar{K}^{0}\right\rangle & =\left\langle 0\left|\bar{s} \gamma^{\eta} \gamma_{5} d\right| K^{0}\right\rangle=-i f_{K} p_{K}^{\eta}, \\
\left\langle 0\left|\bar{d} \gamma_{5} s\right| \bar{K}^{0}\right\rangle & =\left\langle 0\left|\bar{s} \gamma_{5} d\right| K^{0}\right\rangle=i B_{0} f_{K}, \\
\left\langle\pi^{-}\left|\bar{d} \gamma^{\eta} s\right| K^{-}\right\rangle & =\left(p_{K}^{\eta}+p_{\pi}^{\eta}\right) f_{+}+\left(f_{0}-f_{+}\right) q_{K \pi}^{\eta} \frac{m_{K}^{2}-m_{\pi}^{2}}{q_{K \pi}^{2}} \\
\left\langle\pi^{-}|\bar{d} s| K^{-}\right\rangle & =B_{0} f_{0}, \quad q_{K \pi}=p_{K}-p_{\pi}
\end{aligned}
$$

where $f_{K}=155.6(4) \mathrm{MeV}$ [5] is the kaon decay constant and $f_{+, 0}$ are form factors dependent on $q_{K \pi}^{2}$. We also have $\left\langle\pi^{0}\left|\bar{d}\left(\gamma^{\eta}, 1\right) s\right| \bar{K}^{0}\right\rangle=\left\langle\pi^{0}\left|\bar{s}\left(-\gamma^{\eta}, 1\right) d\right| K^{0}\right\rangle=-\left\langle\pi^{-}\right| \bar{d}\left(\gamma^{\eta}\right.$, 1) $s\left|K^{-}\right\rangle / \sqrt{2}$ and $\left\langle\pi^{-}\left|\bar{d} \gamma^{\eta} s\right| K^{-}\right\rangle=\left\langle\pi^{+}\left|\bar{u} \gamma^{\eta} s\right| \bar{K}^{0}\right\rangle$ under the assumption of isospin symmetry. We can then employ $f_{+, 0}=\mathrm{f}_{+}(0)\left(1+\lambda_{+, 0} q_{K \pi}^{2} / m_{\pi^{+}}^{2}\right) \quad$ with $\quad \lambda_{+}=0.0271(10)$ and $\lambda_{0}=0.0142(23)$ from $K_{L} \rightarrow \pi^{+} \mu^{-} \nu$ data [5] and $\mathrm{f}_{+}(0)=0.9681(23)$ from lattice work [25]. For $K^{-} \rightarrow$ $\pi^{0} \pi^{-} \phi \bar{\phi}$ and $K_{L} \rightarrow \pi^{0} \pi^{0} \phi \bar{\phi}$ the pertinent matrix elements are [11]

$$
\begin{aligned}
& \left\langle\pi^{0}\left(p_{0}\right) \pi^{-}\left(p_{-}\right)\left|\bar{d}\left(\gamma^{\eta}, 1\right) \gamma_{5} s\right| K^{-}\right\rangle \\
& \quad=\frac{i \sqrt{2}}{f_{K}}\left[\left(p_{0}^{\eta}-p_{-}^{\eta}, 0\right)+\frac{\left(p_{0}-p_{-}\right) \cdot \tilde{\mathrm{q}}}{m_{K}^{2}-\tilde{\mathrm{q}}^{2}}\left(\tilde{\mathrm{q}}^{\eta},-B_{0}\right)\right], \\
& \left\langle\pi^{0}\left(p_{1}\right) \pi^{0}\left(p_{2}\right)\left|\bar{d}\left(\gamma^{\eta}, 1\right) \gamma_{5} s\right| \bar{K}^{0}\right\rangle \\
& =\frac{i}{f_{K}}\left[\left(p_{1}^{\eta}+p_{2}^{\eta}, 0\right)+\frac{\left(p_{1}+p_{2}\right) \cdot \tilde{\mathrm{q}}}{m_{K}^{2}-\tilde{\mathrm{q}}^{2}}\left(\tilde{\mathrm{q}}^{\eta},-B_{0}\right)\right],
\end{aligned}
$$

where $\tilde{\mathrm{q}}=p_{K^{-}}-p_{0}-p_{-}=p_{\bar{K}^{0}}-p_{1}-p_{2}$. In the $K^{-}$ case, we will drop the small contribution $\left\langle\pi^{0} \pi^{-}\left|\bar{d} \gamma^{\eta} s\right| K^{-}\right\rangle \neq$ 0 arising from the anomaly Lagrangian [17] which is at next-to-leading order in the chiral expansion. We also

ignore form-factor effects in estimating the $K \rightarrow \pi \pi^{\prime} \phi \bar{\phi}$ rates.

From Eq. (9), we obtain the amplitudes for $K_{L, S} \rightarrow \phi \bar{\phi}$ induced by $\mathcal{L}_{\phi}$ to be

$$
\begin{aligned}
& \mathcal{M}_{K_{L} \rightarrow \phi \bar{\phi}}=-\sqrt{2} B_{0} f_{K} \operatorname{Im~c}_{\phi}^{\mathrm{P}}, \\
& \mathcal{M}_{K_{S} \rightarrow \phi \bar{\phi}}=i \sqrt{2} B_{0} f_{K} \operatorname{Rec}_{\phi}^{\mathrm{P}},
\end{aligned}
$$

with the approximate relations $\sqrt{2} K_{L, S}=K^{0} \pm \bar{K}^{0}$. The corresponding decay rates are

$$
\begin{aligned}
\Gamma_{K_{L} \rightarrow \phi \bar{\phi}} & =\frac{B_{0}^{2} f_{K}^{2}}{8 \pi m_{K^{0}}} \sqrt{1-\frac{4 m_{\phi}^{2}}{m_{K^{0}}^{2}}}\left(\operatorname{Im~c}_{\phi}^{\mathrm{P}}\right)^{2}, \\
\Gamma_{K_{S} \rightarrow \phi \bar{\phi}} & =\frac{\left(\operatorname{Rec}_{\phi}^{\mathrm{P}}\right)^{2}}{\left(\operatorname{Im~c}_{\phi}^{\mathrm{P}}\right)^{2}} \Gamma_{K_{L} \rightarrow \phi \bar{\phi}} .
\end{aligned}
$$

For the three-body modes, we derive

$$
\begin{aligned}
& \mathcal{M}_{K^{-} \rightarrow \pi^{-} \phi \bar{\phi}}=2 f_{+} p_{K} \cdot(\mathrm{p}-\overline{\mathrm{p}}) \mathrm{c}_{\phi}^{\mathrm{V}}+B_{0} f_{0} \mathrm{c}_{\phi}^{\mathrm{S}}, \\
& \mathcal{M}_{K_{L} \rightarrow \pi^{0} \phi \bar{\phi}}=-2 i f_{+} p_{K} \cdot(\mathrm{p}-\overline{\mathrm{p}}) \operatorname{Imc}_{\phi}^{\mathrm{V}}-B_{0} f_{0} \operatorname{Rec}_{\phi}^{\mathrm{S}} .
\end{aligned}
$$

These lead to the differential decay rates

$$
\frac{d \Gamma_{K^{-} \rightarrow \pi^{-}} \phi \bar{\phi}}{d \hat{s}}=\frac{\beta \lambda_{K^{-} \pi^{-}}^{1 / 2}}{768 \pi^{3} m_{K^{-}}^{3}}\left(\beta^{2} \lambda_{K^{-} \pi^{-}} f_{+}^{2}\left|\mathbf{c}_{\phi}^{\mathrm{V}}\right|^{2}+3 B_{0}^{2} f_{0}^{2}\left|\mathbf{C}_{\phi}^{\mathrm{S}}\right|^{2}\right),
$$

$$
\frac{d \Gamma_{K_{L} \rightarrow \pi^{0} \phi \bar{\phi}}}{d \hat{s}}=\frac{\beta \lambda_{K^{0} \pi^{0}}^{1 / 2}}{768 \pi^{3} m_{K^{0}}^{3}}\left[\beta^{2} \lambda_{K^{0} \pi^{0}} f_{+}^{2}\left(\operatorname{Imc}_{\phi}^{\mathrm{V}}\right)^{2}+3 B_{0}^{2} f_{0}^{2}\left(\operatorname{Rec}_{\phi}^{S}\right)^{2}\right],
$$

and the rates result from integrating them over $4 m_{\phi}^{2} \leq \hat{s} \leq\left(m_{K^{-}, K^{0}}-m_{\pi^{-}, \pi^{0}}\right)^{2}$, respectively. For the four-body kaon decays, in view of Eq. (10), the amplitudes are

$$
\begin{gathered}
\mathcal{M}_{K^{-} \rightarrow \pi^{0} \pi^{-} \phi \bar{\phi}}=\frac{i \sqrt{2}}{f_{K}}\left[\mathrm{c}_{\phi}^{\mathrm{A}}\left(p_{0}-p_{-}\right) \cdot(\mathrm{p}-\overline{\mathrm{p}})-B_{0} \mathrm{c}_{\phi}^{\mathrm{P}} \frac{\left(p_{0}-p_{-}\right) \cdot \tilde{\mathrm{q}}}{m_{K}^{2}-\hat{s}}\right], \\
\mathcal{M}_{K_{L} \rightarrow \pi^{0} \pi^{0} \phi \bar{\phi}}=\frac{i \sqrt{2}}{f_{K}}\left[\operatorname{Rec}_{\phi}^{\mathrm{A}}\left(p_{1}+p_{2}\right) \cdot(\mathrm{p}-\overline{\mathrm{p}})-i B_{0} \operatorname{Im} \mathrm{c}_{\phi}^{\mathrm{P}} \frac{\left(p_{1}+p_{2}\right) \cdot \tilde{\mathrm{q}}}{m_{K}^{2}-\hat{s}}\right],
\end{gathered}
$$

from which we arrive at the double-differential decay rates ${ }^{3}$

where

$$
\begin{aligned}
& \frac{d^{2} \Gamma_{K^{-} \rightarrow \pi^{0} \pi^{-} \phi \bar{\phi}}}{d \hat{s} d \hat{\varsigma}}=\frac{\beta \beta_{\hat{\zeta}}^{3} \tilde{\lambda}_{K^{-}}^{1 / 2}}{72(4 \pi)^{5} f_{K^{2}}^{2} m_{K^{-}}^{3}}\left[\beta^{2}\left(\tilde{\lambda}_{K^{-}}+12 \hat{s} \hat{\varsigma}\right)\left|\mathbf{c}_{\phi}^{\mathrm{A}}\right|^{2}+\frac{3 \tilde{\lambda}_{K^{-}} B_{0}^{2}}{\left(m_{K}^{2}-\hat{s}\right)^{2}}\left|\mathbf{c}_{\phi}^{\mathrm{P}}\right|^{2}\right], \\
& \frac{d^{2} \Gamma_{K_{L} \rightarrow \pi^{0} \pi^{0} \phi \bar{\phi}}}{d \hat{s} d \hat{\varsigma}}=\frac{\beta \beta_{\hat{\zeta}} \tilde{\lambda}_{K^{0}}^{1 / 2}}{48(4 \pi)^{5} f_{K}^{2} m_{K^{0}}^{3}}\left[\beta^{2} \tilde{\lambda}_{K^{0}}\left(\operatorname{Rec}_{\phi}^{\mathrm{A}}\right)^{2}+\frac{3 B_{0}^{2}\left(m_{K^{0}}^{2}-\hat{s}-\hat{\varsigma}\right)^{2}}{\left(m_{K}^{2}-\hat{s}\right)^{2}}\left(\operatorname{Im~c}{ }_{\phi}^{\mathrm{P}}\right)^{2}\right],
\end{aligned}
$$

\footnotetext{
${ }^{3}$ These formulas in Eq. (19) agree with those given in Ref. [17], except for the overall factor $\beta=\left(1-4 m_{\phi}^{2} / \hat{s}\right)^{1 / 2}$, which appears to be missing from the latter.
} 


$$
\begin{aligned}
& \beta_{\hat{\varsigma}}=\sqrt{1-\frac{4 m_{\pi}^{2}}{\hat{\varsigma}}}, \quad \hat{\varsigma}=\left(p_{0}+p_{-}\right)^{2}=\left(p_{1}+p_{2}\right)^{2}, \\
& \tilde{\lambda}_{K}=\mathcal{K}\left(m_{K}^{2}, \hat{\varsigma}, \hat{\varsigma}\right),
\end{aligned}
$$

and $m_{\pi}$ in the $K^{-}\left(K_{L}\right)$ formula is the isospin-average (neutral) pion mass. Their integration ranges are $4 m_{\phi}^{2} \leq$ $\hat{s} \leq\left(m_{K^{-}, K^{0}}-2 m_{\pi}\right)^{2}$ and $4 m_{\pi}^{2} \leq \hat{\varsigma} \leq\left(m_{K^{-}, K^{0}}-\hat{s}^{1 / 2}\right)^{2}$, respectively.

Evidently, $K_{L, S} \rightarrow \phi \bar{\phi}$ are sensitive to $\mathrm{c}_{\phi}^{\mathrm{P}}$ alone, while $K \rightarrow \pi \pi^{\prime} \phi \bar{\phi}$ and $\Omega^{-} \rightarrow \Xi^{-} \phi \bar{\phi}$ can probe only the two parity-odd coefficients, $\mathrm{C}_{\phi}^{\mathrm{A}}$ and $\mathrm{c}_{\phi}^{\mathrm{P}}$, in our approximation of the hadronic matrix elements. On the other hand, $K \rightarrow$ $\pi \phi \bar{\phi}$ are sensitive to both of the parity-even coefficients, $\mathrm{C}_{\phi}^{\mathrm{V}}$ and $\mathrm{C}_{\phi}^{\mathrm{S}}$, but not to $\mathrm{C}_{\phi}^{\mathrm{A}, \mathrm{P}}$. In contrast, the spin- $1 / 2$ hyperon modes $\mathfrak{B} \rightarrow \mathfrak{B}^{\prime} \phi \bar{\phi}$ can probe all the coefficients, according to Eq. (7). It follows that quests for $\mathfrak{B} \rightarrow \mathfrak{B}^{\prime} \mathbb{E}$ as well as $\Omega^{-} \rightarrow \Xi^{-} \not E$ are beneficial because the acquired data could reveal information on $d s \phi \phi$ interactions which complements that gained from kaon measurements. Furthermore, a couple of the hyperon modes, namely, $\Sigma^{+} \rightarrow p \phi \bar{\phi}$ and $\Omega^{-} \rightarrow \Xi^{-} \phi \bar{\phi}$, can cover broader $m_{\phi}$ ranges than $K \rightarrow$ $\pi \pi^{\prime} \phi \bar{\phi}$ and therefore provide the only window into $\mathrm{C}_{\phi}^{\mathrm{A}}$ for certain $m_{\phi}$ values, as we discuss later on.

\section{NUMERICAL RESULTS}

Although there is still no empirical information on the hyperon decays with missing energy, their first data may become available from BESIII not long from now. Their branching fractions in the SM have been estimated to be less than $10^{-11}$ in Ref. [11] and hence are several orders of magnitude below the estimated sensitivity levels of BESIII [9], as can be viewed in Table I. Although the SM numbers are unlikely to be probed in the near future, NP effects turn out to be presently allowed to boost the branching fractions tremendously to values which may be testable by BESIII.

Among their kaon counterparts, the data on $K \rightarrow \pi \mathscr{E}$ are the closest to their SM expectations. Particularly, the E949
[2] and KOTO [4] findings, $\mathcal{B}\left(K^{+} \rightarrow \pi^{+} \nu \nu\right)=1.7(1.1) \times$ $10^{-10}$ [5] and $\mathcal{B}\left(K_{L} \rightarrow \pi^{0} \nu \bar{\nu}\right)<3.0 \times 10^{-9}$ at $90 \%$ confidence level (C.L.), respectively, are not far from the SM numbers $\mathcal{B}\left(K^{+} \rightarrow \pi^{+} \nu \nu\right)=\left(8.5_{-1.2}^{+1.0}\right) \times 10^{-11}$ and $\mathcal{B}\left(K_{L} \rightarrow\right.$ $\left.\pi^{0} \nu \bar{\nu}\right)=\left(3.2_{-0.7}^{+1.1}\right) \times 10^{-11}$ [26]. We can regard the upper ends of the $90 \%$-C.L. ranges of their deviations from the SM predictions,

$$
\begin{aligned}
\Delta \mathcal{B}\left(K^{-} \rightarrow \pi^{-} \not E\right) & <2.7 \times 10^{-10}, \\
\Delta \mathcal{B}\left(K_{L} \rightarrow \pi^{0} \not E\right) & <3.0 \times 10^{-9},
\end{aligned}
$$

as capping the NP contributions. These conditions prevent $\mathrm{C}_{\phi}^{\mathrm{V}, \mathrm{S}}$ from being sizable.

By contrast, there is still comparatively greater room for NP to impact $K \rightarrow \mathscr{E}$ and $K \rightarrow \pi \pi^{\prime} \mathbb{E}$. For the former, although direct searches are yet to be conducted [5], there are indirect upper bounds (at 95\% C.L.) extracted from the existing data on $K_{L, S}$ visible decay channels [8]:

$$
\begin{aligned}
\mathcal{B}\left(K_{L} \rightarrow \not{E}\right)<6.3 \times 10^{-4}, \\
\mathcal{B}\left(K_{S} \rightarrow \not{E}\right)<1.1 \times 10^{-4} .
\end{aligned}
$$

These numbers are way higher than $\mathcal{B}\left(K_{L} \rightarrow \nu \bar{\nu}\right) \lesssim 1 \times 10^{-10}$ and $\mathcal{B}\left(K_{S} \rightarrow \nu \bar{\nu}\right) \lesssim 2 \times 10^{-14}$ estimated in the SM supplemented with neutrino mass $[8,11] .{ }^{4}$ For the four-body modes, the empirical limits $\mathcal{B}\left(K^{-} \rightarrow \pi^{0} \pi^{-} \nu \bar{\nu}\right)<4.3 \times 10^{-5}$ [6] and $\mathcal{B}\left(K_{L} \rightarrow \pi^{0} \pi^{0} \nu \bar{\nu}\right)<8.1 \times 10^{-7}$ [7] are the only data available and again vastly exceed the SM expectations of order $10^{-14}$ and $10^{-13}$, respectively [17,28,29]. Accordingly, we may impose

$$
\begin{aligned}
\mathcal{B}\left(K^{-} \rightarrow \pi^{0} \pi^{-} \not E\right) & <4 \times 10^{-5}, \\
\mathcal{B}\left(K_{L} \rightarrow \pi^{0} \pi^{0} \not \mathcal{E}\right) & <8 \times 10^{-7}
\end{aligned}
$$

on NP contributions.

To explore how much NP can enhance the hyperon rates, we consider first the possibility that the $\phi$ mass can be ignored relative to the pion mass or vanishes, $m_{\phi}=0$. In this case, integrating the differential rates of the baryon decays, we arrive at the branching fractions

$$
\begin{aligned}
\mathcal{B}(\Lambda \rightarrow n \phi \bar{\phi}) & =\left[0.11\left|\mathrm{c}_{\phi}^{\mathrm{V}}\right|^{2}+0.18\left|\mathrm{c}_{\phi}^{\mathrm{A}}\right|^{2}+\left(42\left|\mathrm{c}_{\phi}^{\mathrm{S}}\right|^{2}+12\left|\mathrm{c}_{\phi}^{\mathrm{P}}\right|^{2}\right) \mathrm{GeV}^{-2}\right] 10^{8} \mathrm{GeV}^{4}, \\
\mathcal{B}\left(\Sigma^{+} \rightarrow p \phi \bar{\phi}\right) & =\left[0.13\left|\mathrm{c}_{\phi}^{\mathrm{V}}\right|^{2}+0.046\left|\mathrm{c}_{\phi}^{\mathrm{A}}\right|^{2}+\left(49\left|\mathrm{c}_{\phi}^{\mathrm{S}}\right|^{2}+3.4\left|\mathrm{c}_{\phi}^{\mathrm{P}}\right|^{2}\right) \mathrm{GeV}^{-2}\right] 10^{8} \mathrm{GeV}^{4}, \\
\mathcal{B}\left(\Xi^{0} \rightarrow \Lambda \phi \bar{\phi}\right) & =\left[0.23\left|\mathrm{c}_{\phi}^{\mathrm{V}}\right|^{2}+0.025\left|\mathrm{c}_{\phi}^{\mathrm{A}}\right|^{2}+\left(91\left|\mathrm{c}_{\phi}^{\mathrm{S}}\right|^{2}+1.8\left|\mathrm{c}_{\phi}^{\mathrm{P}}\right|^{2}\right) \mathrm{GeV}^{-2}\right] 10^{8} \mathrm{GeV}^{4}, \\
\mathcal{B}\left(\Xi^{0} \rightarrow \Sigma^{0} \phi \bar{\phi}\right) & =\left[0.07\left|\mathrm{c}_{\phi}^{\mathrm{V}}\right|^{2}+0.34\left|\mathrm{c}_{\phi}^{\mathrm{A}}\right|^{2}+\left(28\left|\mathrm{c}_{\phi}^{\mathrm{S}}\right|^{2}+23\left|\mathrm{c}_{\phi}^{\mathrm{P}}\right|^{2}\right) \mathrm{GeV}^{-2}\right] 10^{7} \mathrm{GeV}^{4}, \\
\mathcal{B}\left(\Xi^{-} \rightarrow \Sigma^{-} \phi \bar{\phi}\right) & =\left[0.09\left|\mathrm{c}_{\phi}^{\mathrm{V}}\right|^{2}+0.42\left|\mathrm{c}_{\phi}^{\mathrm{A}}\right|^{2}+\left(33\left|\mathrm{c}_{\phi}^{\mathrm{S}}\right|^{2}+28\left|\mathrm{c}_{\phi}^{\mathrm{P}}\right|^{2}\right) \mathrm{GeV}^{-2}\right] 10^{7} \mathrm{GeV}^{4},
\end{aligned}
$$

\footnotetext{
${ }^{4}$ These results follow from the use of the neutrino mass' highest direct limit $m_{\nu_{\tau}}^{\exp }<18.2 \mathrm{MeV}$ listed by the Particle Data Group [5]. If one employs instead the cosmological bound of under $1 \mathrm{eV}$ for the sum of neutrino masses [5], the invisible widths of $K_{L, S}$ turn out to be dominated by their decays into four neutrinos with branching fractions below $10^{-21}$ and $10^{-24}$, respectively [27].
} 
TABLE I. The branching fractions of $|\Delta S|=1$ hyperon decays with missing energy in the SM [11] and the corresponding expected sensitivities of BESIII [9].

\begin{tabular}{lcccccc}
\hline \hline Decay mode & $\Lambda \rightarrow n \nu \bar{\nu}$ & $\Sigma^{+} \rightarrow p \nu \bar{\nu}$ & $\Xi^{0} \rightarrow \Lambda \nu \bar{\nu}$ & $\Xi^{0} \rightarrow \Sigma^{0} \nu \bar{\nu}$ & $\Xi^{-} \rightarrow \Sigma^{-} \nu \bar{\nu}$ & $\Omega^{-} \rightarrow \Xi^{-} \nu \bar{\nu}$ \\
\hline SM branching fraction [11] & $7.1 \times 10^{-13}$ & $4.3 \times 10^{-13}$ & $6.3 \times 10^{-13}$ & $1.0 \times 10^{-13}$ & $1.3 \times 10^{-13}$ & $4.9 \times 10^{-12}$ \\
Expected BESIII sensitivity [9] & $3 \times 10^{-7}$ & $4 \times 10^{-7}$ & $8 \times 10^{-7}$ & $9 \times 10^{-7}$ & $\cdots$ & $2.6 \times 10^{-5}$ \\
\hline \hline
\end{tabular}

$\mathcal{B}\left(\Omega^{-} \rightarrow \Xi^{-} \phi \bar{\phi}\right)=\left(2.0\left|\mathrm{c}_{\phi}^{\mathrm{A}}\right|^{2}+152\left|\mathrm{c}_{\phi}^{\mathrm{P}}\right|^{2} \mathrm{GeV}^{-2}\right) 10^{8} \mathrm{GeV}^{4}$

All these results have incorporated the form factors mentioned in Sec. III A.

As mentioned above, the current $K \rightarrow \pi \nu \bar{\nu}$ data do not leave ample room for NP to affect $c_{\phi}^{\mathrm{V}, \mathrm{S}}$ significantly. More specifically, if these coefficients are the only ones being nonzero, comparing their contributions, for $m_{\phi}=0$,

$$
\begin{aligned}
& \mathcal{B}\left(K^{-} \rightarrow \pi^{-} \phi \bar{\phi}\right) \\
& \quad=\left(0.033\left|\mathrm{c}_{\phi}^{\mathrm{V}}\right|^{2}+14\left|\mathrm{c}_{\phi}^{\mathrm{S}}\right|^{2} \mathrm{GeV}^{-2}\right) 10^{11} \mathrm{GeV}^{4} \\
& \mathcal{B}\left(K_{L} \rightarrow \pi^{0} \phi \bar{\phi}\right) \\
& \quad=\left[0.15\left(\operatorname{Imc}_{\phi}^{\mathrm{V}}\right)^{2}+59\left(\operatorname{Rec}_{\phi}^{\mathrm{S}}\right)^{2} \mathrm{GeV}^{-2}\right] 10^{11} \mathrm{GeV}^{4}
\end{aligned}
$$

with the corresponding bounds in Eq. (21), we find that the resulting hyperon branching fractions in Eqs. (24) and (25) are below $10^{-11}$. This scenario would therefore be out of BESIII reach according to Table I.

Therefore, hereafter we concentrate on the possibility that NP can generate considerable effects solely through $\mathrm{C}_{\phi}^{\mathrm{A}, \mathrm{P}}$. With this assumption and $m_{\phi}=0$, for the two-body kaon decays we get

$$
\begin{aligned}
& \mathcal{B}\left(K_{L} \rightarrow \phi \bar{\phi}\right)=5.9 \times 10^{14} \mathrm{GeV}^{2}\left(\operatorname{Im~c}_{\phi}^{\mathrm{P}}\right)^{2}, \\
& \mathcal{B}\left(K_{S} \rightarrow \phi \bar{\phi}\right)=1.0 \times 10^{12} \mathrm{GeV}^{2}\left(\operatorname{Re} \mathrm{C}_{\phi}^{\mathrm{P}}\right)^{2},
\end{aligned}
$$

implying

$$
\begin{aligned}
\left|\mathrm{c}_{\phi}^{\mathrm{P}}\right|^{2}= & \frac{1.7 \times 10^{-15}}{\mathrm{GeV}^{2}} \mathcal{B}\left(K_{L} \rightarrow \phi \bar{\phi}\right) \\
& +\frac{9.7 \times 10^{-13}}{\mathrm{GeV}^{2}} \mathcal{B}\left(K_{S} \rightarrow \phi \bar{\phi}\right),
\end{aligned}
$$

and for the four-body decays

$$
\begin{aligned}
& \mathcal{B}\left(K^{-} \rightarrow \pi^{-} \pi^{0} \phi \bar{\phi}\right) \\
& \quad=\left(0.16\left|\mathrm{c}_{\phi}^{\mathrm{A}}\right|^{2}+14\left|\mathrm{c}_{\phi}^{\mathrm{P}}\right|^{2} \mathrm{GeV}^{-2}\right) 10^{6} \mathrm{GeV}^{4}, \\
& \mathcal{B}\left(K_{L} \rightarrow \pi^{0} \pi^{0} \phi \bar{\phi}\right) \\
& \quad=\left[0.21\left(\operatorname{Re} \mathrm{c}_{\phi}^{\mathrm{A}}\right)^{2}+64\left(\mathrm{Im} \mathrm{c}_{\phi}^{\mathrm{P}}\right)^{2} \mathrm{GeV}^{-2}\right] 10^{7} \mathrm{GeV}^{4} .
\end{aligned}
$$

To illustrate the potential impact of sizable $\mathrm{c}_{\phi}^{\mathrm{A}, \mathrm{P}}$ on the hyperon modes, we can look at a couple of examples with different choices of the nonvanishing coupling, which we take to be real to avoid any new $C P$-violation source.

Thus, if only $\mathrm{c}_{\phi}^{\mathrm{P}} \neq 0$, the $K \rightarrow \mathscr{E}$ restraints in Eq. (22) turn out to be more stringent than the $K \rightarrow \pi \pi^{\prime} \mathbb{E}$ ones in Eq. (23) and, with the aid of Eq. (29), lead to $\left|\mathbf{c}_{\phi}^{\text {P }}\right|^{2}<$ $1.1 \times 10^{-16} \mathrm{GeV}^{-2}$. Plugging this into Eqs. (24)-(26) and setting $\mathrm{c}_{\phi}^{\mathrm{V}, \mathrm{A}, \mathrm{S}}=0$, we obtain

$$
\begin{aligned}
\mathcal{B}(\Lambda \rightarrow n \phi \bar{\phi}) & <1.3 \times 10^{-7}, \\
\mathcal{B}\left(\Sigma^{+} \rightarrow p \phi \bar{\phi}\right) & <3.7 \times 10^{-8}, \\
\mathcal{B}\left(\Xi^{0} \rightarrow \Lambda \phi \bar{\phi}\right) & <1.9 \times 10^{-8}, \\
\mathcal{B}\left(\Xi^{0} \rightarrow \Sigma^{0} \phi \bar{\phi}\right) & <2.5 \times 10^{-8}, \\
\mathcal{B}\left(\Xi^{-} \rightarrow \Sigma^{-} \phi \bar{\phi}\right) & <3.0 \times 10^{-8}, \\
\mathcal{B}\left(\Omega^{-} \rightarrow \Xi^{-} \phi \bar{\phi}\right) & <1.6 \times 10^{-6} .
\end{aligned}
$$

The upper ends of these ranges greatly exceed the corresponding SM values quoted in Table I but are still below the expected BESIII sensitivity levels, mostly by at least an order of magnitude.

If instead $\mathrm{c}_{\phi}^{\mathrm{A}}$ is nonvanishing and real, whereas $\mathrm{c}_{\phi}^{\mathrm{V}, \mathrm{P}, \mathrm{S}}=$ 0 , then the $K \rightarrow E$ bounds do not matter any more but the $K \rightarrow \pi \pi^{\prime} \mathscr{E}$ ones in Eq. (23) still do, the $K_{L} \rightarrow \pi^{0} \pi^{0} \mathscr{E}$ one being the stronger and yielding $\left(\operatorname{Rec}_{\phi}^{A}\right)^{2}<3.8 \times$ $10^{-13} \mathrm{GeV}^{-4}$. This now translates into

$$
\begin{aligned}
\mathcal{B}(\Lambda \rightarrow n \phi \bar{\phi}) & <6.6 \times 10^{-6}, \\
\mathcal{B}\left(\Sigma^{+} \rightarrow p \phi \bar{\phi}\right) & <1.7 \times 10^{-6}, \\
\mathcal{B}\left(\Xi^{0} \rightarrow \Lambda \phi \bar{\phi}\right) & <9.4 \times 10^{-7}, \\
\mathcal{B}\left(\Xi^{0} \rightarrow \Sigma^{0} \phi \bar{\phi}\right) & <1.3 \times 10^{-6}, \\
\mathcal{B}\left(\Xi^{-} \rightarrow \Sigma^{-} \phi \bar{\phi}\right) & <1.6 \times 10^{-6}, \\
\mathcal{B}\left(\Omega^{-} \rightarrow \Xi^{-} \phi \bar{\phi}\right) & <7.5 \times 10^{-5}
\end{aligned}
$$

Their upper values are larger than the corresponding BESIII sensitivity levels quoted in Table I. Allowing $\operatorname{Im~} \mathrm{c}_{\phi}^{\mathrm{A}} \neq 0$, one could get higher branching fractions with $\mathrm{C}_{\phi}^{\mathrm{A}}$ being complex, as its imaginary part would escape the $K_{L} \rightarrow$ $\pi^{0} \pi^{0} \mathbb{E}$ restriction and be subject only to the milder $K^{-} \rightarrow$ $\pi^{0} \pi^{-} \mathscr{E}$ one. The numbers in Eq. (32) are the same as their counterparts in the case treated in Ref. [11] where the invisible particles are Dirac spin-1/2 fermions, highlighting the similarities of the two scenarios when the masses of the invisible particles can be neglected. 


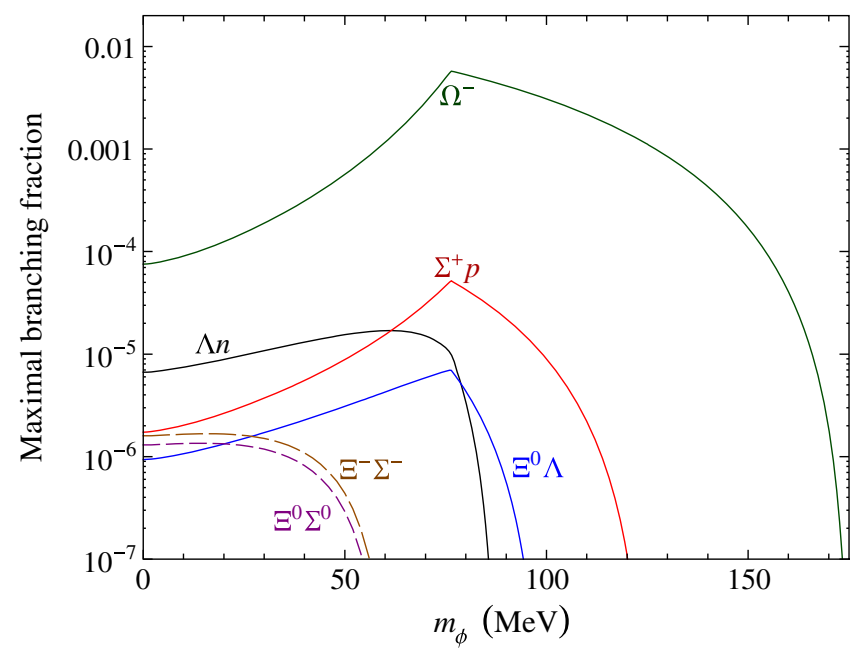

FIG. 1. The maximal branching fractions of $\mathfrak{B} \rightarrow \mathfrak{B}^{\prime} \phi \bar{\phi}$ with $\mathfrak{B} \mathfrak{B}^{\prime}=\Lambda n, \Sigma^{+} p, \Xi^{0} \Lambda, \Xi^{0} \Sigma^{0}, \Xi^{-} \Sigma^{-}$and of $\Omega^{-} \rightarrow \Xi^{-} \phi \bar{\phi}$, indicated on the plot by the $\mathfrak{B B}^{\prime}$ and $\Omega^{-}$labels, respectively, versus $m_{\phi}$, induced by the contribution of $\operatorname{Re}_{\phi}^{A}$ alone, subject to the $K_{L} \rightarrow \pi^{0} \pi^{0} E$ constraint and the perturbativity requirement for $m_{\phi}>76 \mathrm{MeV}$ as explained in the text.

We consider next the possibility that $m_{\phi}$ is nonnegligible and let it vary over the allowed kinematical range of each of the hyperon decays. As in the second example, we suppose that $\mathrm{C}_{\phi}^{\mathrm{A}}$ is nonzero and real, while $\mathrm{c}_{\phi}^{\mathrm{V}, \mathrm{S}, \mathrm{P}}=0$, and so the $K_{L} \rightarrow \pi^{0} \pi^{0} \mathbb{E}$ restriction again needs to be taken into account. Now, as $m_{\phi}$ grows this restriction increasingly weakens until it no longer applies at $m_{\phi} \simeq 114 \mathrm{MeV}$ when $K_{L} \rightarrow \pi^{0} \pi^{0} \mathbb{E}$ becomes kinematically closed. Accordingly, for $m_{\phi}>0$ the hyperon rates can be substantially bigger

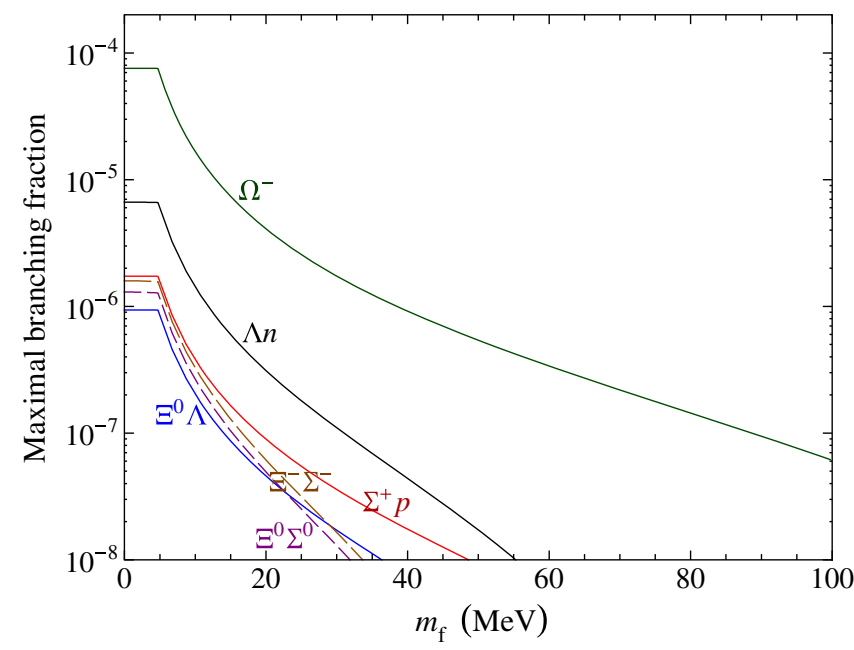

FIG. 2. The maximal branching fractions of $\mathfrak{B} \rightarrow \mathfrak{B}^{\prime} £ \overline{\mathrm{f}}$ with $\mathfrak{B} \mathfrak{B}^{\prime}=\Lambda n, \Sigma^{+} p, \Xi^{0} \Lambda, \Xi^{0} \Sigma^{0}, \Xi^{-} \Sigma^{-}$and of $\Omega^{-} \rightarrow \Xi^{-} \mathrm{f} \overline{\mathrm{f}}$ versus $m_{\mathrm{f}}$, induced by the contribution of $\operatorname{Re} \tilde{\mathrm{C}}_{\mathrm{f}}^{\mathrm{A}}$ alone, subject to the $K_{L} \rightarrow \pi^{0} \pi^{0} \mathbb{E}$ and $K_{L} \rightarrow \mathbb{E}$ constraints, with the latter becoming more important for $m_{\mathrm{f}}>5 \mathrm{MeV}$.
TABLE II. New-physics couplings contributing to $K \rightarrow \mathbb{E}$ and $K \rightarrow \pi \pi^{\prime} \mathscr{E}$ if $\mathscr{E}$ is carried away by spin-0 bosons $\phi \bar{\phi}$ or Dirac spin- $1 / 2$ fermions $\mathrm{f} \overline{\mathrm{f}}$ and their masses are nonzero, $m_{\phi, \mathrm{f}}>0$. All these couplings belong to operators involving parity-odd $d s$ quark bilinears.

\begin{tabular}{lcccc}
\hline \hline Kaon mode & $K \rightarrow \phi \bar{\phi}$ & $K \rightarrow \pi \pi^{\prime} \phi \bar{\phi}$ & $K \rightarrow \mathrm{f} \overline{\mathrm{f}}$ & $K \rightarrow \pi \pi^{\prime} \mathrm{f} \overline{\mathrm{f}}$ \\
\hline Couplings & $\mathrm{c}_{\phi}^{\mathrm{P}}$ & $\mathrm{C}_{\phi}^{\mathrm{A}}, \mathrm{c}_{\phi}^{\mathrm{P}}$ & $\tilde{\mathrm{c}}_{\mathrm{f}}^{\mathrm{A}}, \tilde{\mathrm{c}}_{\mathrm{f}}^{\mathrm{S}}, \tilde{\mathrm{c}}_{\mathrm{f}}^{\mathrm{P}}$ & $\tilde{\mathrm{c}}_{\mathrm{f}}^{\mathrm{V}}, \tilde{\mathrm{c}}_{\mathrm{f}}^{\mathrm{A}}, \tilde{\mathrm{c}}_{\mathrm{f}}^{\mathrm{S}}, \tilde{\mathrm{c}}_{\mathrm{f}}^{\mathrm{P}}$ \\
\hline \hline
\end{tabular}

than their $m_{\phi}=0$ values, and for $m_{\phi}>114 \mathrm{MeV}$ the kaon decay constraint is no more. However, as the restraint on $\left|\operatorname{Rec}_{\phi}^{\mathrm{A}}\right|$ continues to loosen, its rising upper bound may no longer be compatible with the perturbativity of the $\phi$ interactions. To ensure that they remain perturbative, inspired by the form of the standard effective weak interaction we impose $\left|\operatorname{Rec}_{\phi}^{A}\right|<G_{\mathrm{F}} / \sqrt{2} \simeq 8.2 / \mathrm{TeV}^{2}$, where $G_{\mathrm{F}}$ is the Fermi constant. Since this corresponds to the requisite $\mathcal{B}\left(K_{L} \rightarrow \pi^{0} \pi^{0} \phi \bar{\phi}\right)<8 \times 10^{-7}$ at $m_{\phi} \simeq 76 \mathrm{MeV}$, we then set $\left|\operatorname{Re} \mathrm{C}_{\phi}^{\mathrm{A}}\right|=G_{\mathrm{F}} / \sqrt{2}$ for $m_{\phi}>76 \mathrm{MeV}$. In Fig. 1 we depict the resulting maximal branching fractions of the hyperon modes versus $m_{\phi}$ varying from 0 to $175 \mathrm{MeV}$, which is approximately its highest value in $\Omega^{-} \rightarrow \Xi^{-} \phi \bar{\phi}$. Evidently, over the majority of their respective $m_{\phi}$ ranges, four of the modes (the ones with $\mathfrak{B B}^{\prime}=\Lambda n, \Sigma^{+} p, \Xi^{0} \Lambda$ and the $\Omega^{-}$one) have maximal branching fractions that are significantly greater than their $m_{\phi}=0$ values, implying that their $m_{\phi}>0$ predictions are easier to test experimentally.

Now, in the $s \rightarrow d \mathbb{E}$ scenario explored in Ref. [11] the invisible particles are Dirac spin- $1 / 2$ fermions, $f \bar{f}$, and the numerical work therein focused on instances where the f mass, $m_{\mathrm{f}}$, was negligible or zero. Here we look at what may happen if $m_{\mathrm{f}}$ is not negligible. In that case, as Table II summarizes, $K \rightarrow \mathrm{f} \overline{\mathrm{f}}$ and $K \rightarrow \pi \pi^{\prime} \mathrm{f} \overline{\mathrm{f}}$ receive contribu-

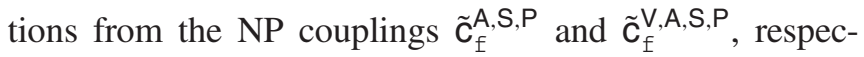
tively, which parametrize the effective $d s f f$ interactions described by the Lagrangian $\mathcal{L}_{\mathrm{f}} \supset-\bar{d} \gamma^{\eta} \gamma_{5} s \overline{\mathrm{f}} \gamma_{\eta}\left(\tilde{\mathrm{c}}_{\mathrm{f}}^{\mathrm{V}}+\right.$ $\left.\gamma_{5} \tilde{\mathrm{C}}_{\mathrm{f}}^{\mathrm{A}}\right) \mathrm{f}-\bar{d} \gamma_{5} s \overline{\mathrm{f}}\left(\tilde{\mathrm{C}}_{\mathrm{f}}^{\mathrm{S}}+\gamma_{5} \tilde{\mathrm{C}}_{\mathrm{f}}^{\mathrm{P}}\right) \mathrm{f}+$ H.c. used in Ref. [11]. ${ }^{5}$ Consequently, for $\tilde{\mathrm{C}}_{\mathrm{f}}^{\mathrm{A}, \mathrm{S}, \mathrm{P}}$ the $K \rightarrow \mathbb{E}$ restrictions become more important than the $K \rightarrow \pi \pi^{\prime} \mathscr{E}$ ones over most of the $m_{\mathrm{f}}>0$ region, with the implication that the resulting hyperon rates tend to be much smaller than their $m_{\mathrm{f}}=0$ values. This is illustrated in Fig. 2, for which $\operatorname{Re} \tilde{\mathrm{C}}_{\mathrm{f}}^{\mathrm{A}}$ is taken to be the only coupling being present. From the figure, we learn that the $K_{L} \rightarrow \mathbb{E}$ constraint is stricter than the $K_{L} \rightarrow \pi^{0} \pi^{0} E$ one if $m_{\mathrm{f}}>5 \mathrm{MeV}$. This plot also reveals striking differences from Fig. 1 when the invisible particle's mass is not negligible.

\footnotetext{
${ }^{5}$ In Ref. [11], an overall factor of $\beta=\sqrt{1-4 m_{\mathrm{f}}^{2} / \hat{s}}$ is missing from both Eqs. (B.3) and (B.4) for the double-differential rates of $K^{-} \rightarrow \pi^{0} \pi^{-}$f而 and $K_{L} \rightarrow \pi^{0} \pi^{0} \mathrm{f} \overline{\mathrm{f}}$. It appears to be missing also from the corresponding formulas in Ref. [17].
} 


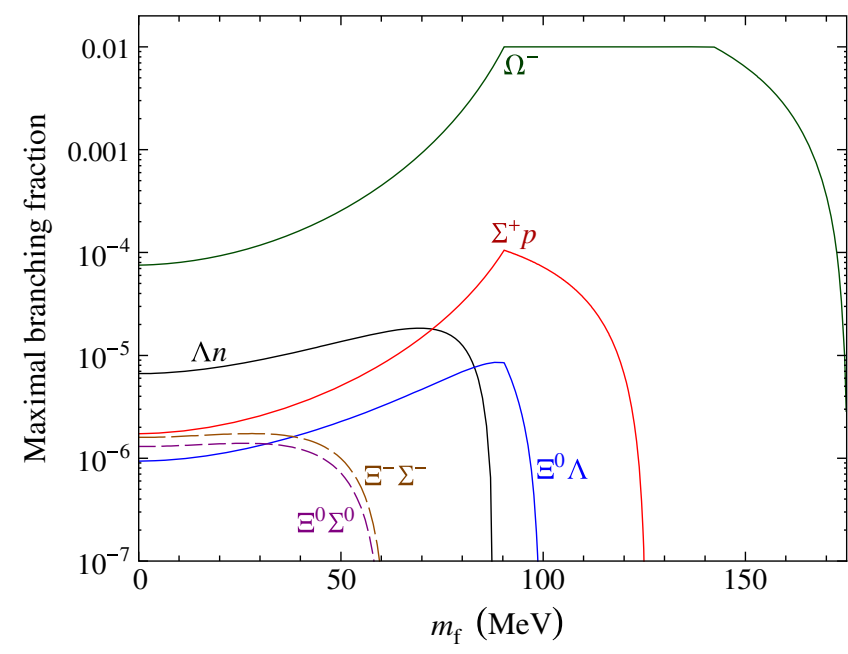

FIG. 3. The maximal branching fractions of $\mathfrak{B} \rightarrow \mathfrak{B}^{\prime} \pm \bar{f}$ with $\mathfrak{B} \mathfrak{B}^{\prime}=\Lambda n, \Sigma^{+} p, \Xi^{0} \Lambda, \Xi^{0} \Sigma^{0}, \Xi^{-} \Sigma^{-}$and of $\Omega^{-} \rightarrow \Xi^{-} \mathrm{f} \overline{\mathrm{f}}$ versus $m_{\mathrm{f}}$, induced by the contribution of $\operatorname{Re} \tilde{\mathrm{C}}_{f}^{V}$ alone, subject to the $K_{L} \rightarrow \pi^{0} \pi^{0} \mathscr{E}$ constraint and the perturbativity and $\Omega^{-}$data requirements for $m_{\mathrm{f}}>90 \mathrm{MeV}$ as explained in the text.

If instead only $\operatorname{Re} \tilde{C}_{f}^{V}$ is nonvanishing, then it evades the $K \rightarrow \mathbb{E}$ restrictions completely and is subject only to the $K \rightarrow \pi \pi^{\prime} \mathbb{E}$ ones, similarly to the bosonic scenario with $\mathrm{C}_{\phi}^{\mathrm{A}}$ being the only coupling, as can be viewed also from Table II. We again demand $\left|\operatorname{Re} \tilde{c}_{f}^{\mathrm{V}}\right|<G_{\mathrm{F}} / \sqrt{2}$ to satisfy the perturbativity requisite, which in this case applies for $m_{\mathrm{f}}>96 \mathrm{MeV}$. However, under these conditions we find that $\operatorname{Re} \tilde{c}_{f}^{V}$ for $m_{\mathrm{f}} \in[90,142] \mathrm{MeV}$ translates into upper bounds on $\mathcal{B}\left(\Omega^{-} \rightarrow \Xi^{-} \mathrm{f} \overline{\mathrm{f}}\right)$ that exceed the allowed value of roughly $1 \%$ for the unknown $\Omega^{-}$decay channels, which is inferred from the data on the observed ones [5]. Therefore, for this $m_{\mathrm{f}}$ region we additionally require $\operatorname{Re} \tilde{C}_{f}^{V}$ to yield $\mathcal{B}\left(\Omega^{-} \rightarrow \Xi^{-} f \bar{f}\right)<1 \%$. We display the resulting branching-fraction limits of the various hyperon modes in Fig. 3 which shows stark differences from Fig. 2 for $m_{\mathrm{f}}>5 \mathrm{MeV}$. On the other hand, Fig. 3 resembles Fig. 1 in important ways, especially in their depictions of the maximal branching fractions which are hugely amplified over much of the $m_{\phi, \mathrm{f}}>0$ ranges relative to their values at $m_{\phi, \mathrm{f}}=0$. There are also dissimilarities between Figs. 1 and 3, one of which is that the curves in the latter tend to be higher at larger masses.

Finally, we note that for $\mathrm{f}$ being a Majorana, rather than Dirac, fermion the $d s \mathrm{ff}$ operators involving the vector bilinear of $f$ do not exist, as its Majorana nature implies $\overline{\mathrm{f}} \gamma^{\mu} \mathrm{f}=0$ [30]. In other words, the contribution of $\tilde{\mathrm{c}}_{\mathrm{f}}^{\mathrm{V}}$ (and of $\mathrm{C}_{\mathrm{f}}^{\mathrm{V}}$, which belongs to the $\bar{d} \gamma^{\eta} s \overline{\mathrm{f}} \gamma_{\eta} \mathrm{f}$ operator [11]) is absent if $\mathrm{f}$ is a Majorana particle. It follows that in this case the substantial increases of the maximal branching fractions like those seen in Fig. 3 do not occur and instead, if significant NP affects mostly $\operatorname{Re} \tilde{\mathrm{C}}_{\mathrm{f}}^{\mathrm{A}}$, the situation is roughly similar to that pictured in Fig. 2.

\section{CONCLUSIONS}

We have explored the strangeness-changing decays of hyperons into another baryon plus missing energy carried away by a pair of invisible spinless bosons, $\phi \bar{\phi}$, which are SM gauge singlet. These processes arise from their effective interactions with the $d$ and $s$ quarks at low energies. Adopting a model-independent approach, we start from an effective Lagrangian respecting SM gauge invariance and containing dimension-six $d s \phi \phi$ operators. Thus, they contribute also to FCNC kaon decays with missing energy and are subject to restrictions from their data. Although the existing $K \rightarrow \pi \mathbb{E}$ constraints do not permit NP to generate sizable effects via parity-even $d s \phi \phi$ operators, we demonstrate that the restrictions from $K \rightarrow \mathbb{E}$ and $K \rightarrow \pi \pi^{\prime} \mathbb{E}$ are much weaker on the parity-odd operators. Specifically, the one with the axial-vector quark bilinear needs to fulfill only the mild $K \rightarrow \pi \pi^{\prime} \mathbb{E}$ restraints and could give rise to considerable hyperon rates which may be within the reach of BESIII or future measurements. Moreover, if NP enters predominantly through the coefficient $\mathrm{C}_{\phi}^{\mathrm{A}}$ of this operator and $m_{\phi}>0$, the kaon decay constraints become weakened and as a result the hyperon rates may be greatly enhanced with respect to their $m_{\phi}=0$ predictions. Interestingly, these kaon constraints on $\mathrm{c}_{\phi}^{\mathrm{A}}$ are absent if $m_{\phi}>114 \mathrm{MeV}$ but two of the hyperon modes, especially $\Omega^{-} \rightarrow \Xi^{-} \phi \bar{\phi}$, can still serve as direct probes of $\mathrm{C}_{\phi}^{\mathrm{A}}$ until $m_{\phi}$ reaches $175 \mathrm{MeV}$. We also perform comparisons with the scenario in which the invisible particles are Dirac spin- $1 / 2$ fermions. We discuss special instances in which the bosonic and fermionic cases may be discriminated experimentally, particularly if their masses are nonvanishing. The results of our analysis illustrate well that these rare hyperon decays and their kaon counterparts are complementary to each other in providing access to possible NP in $s \rightarrow d \mathbb{E}$ transitions.

\section{ACKNOWLEDGMENTS}

This research was supported in part by the MOST (Grant No. MOST 106-2112-M-002-003-MY3). 
[1] G. Buchalla, A. J. Buras, and M. E. Lautenbacher, Weak decays beyond leading logarithms, Rev. Mod. Phys. 68, 1125 (1996).

[2] A. V. Artamonov et al. (E949 Collaboration), New Measurement of the $K^{+} \rightarrow \pi^{+} \nu \bar{\nu}$ Branching Ratio, Phys. Rev. Lett. 101, 191802 (2008).

[3] E. Cortina Gil et al. (NA62 Collaboration), First search for $K^{+} \rightarrow \pi^{+} \nu \bar{\nu}$ using the decay-in-flight technique, Phys. Lett. B 791, 156 (2019).

[4] J. K. Ahn et al. (KOTO Collaboration), Search for the $K_{L} \rightarrow$ $\pi^{0} \nu \bar{\nu}$ and $K_{L} \rightarrow \pi^{0} X^{0}$ Decays at the J-PARC KOTO Experiment, Phys. Rev. Lett. 122, 021802 (2019).

[5] M. Tanabashi et al. (Particle Data Group), Review of particle physics, Phys. Rev. D 98, 030001 (2018).

[6] S. Adler et al. (E787 Collaboration), Search for the decay $K^{+} \rightarrow \pi^{+} \pi^{0} \nu \bar{\nu}$, Phys. Rev. D 63, 032004 (2001).

[7] R. Ogata et al. (E391a Collaboration), Study of the $K_{L}^{0} \rightarrow$ $\pi^{0} \pi^{0} \nu \bar{\nu}$ decay, Phys. Rev. D 84, 052009 (2011).

[8] S. N. Gninenko, Search for invisible decays of $\pi^{0}, \eta, \eta^{\prime}, K_{S}$ and $K_{L}$ : A probe of new physics and tests using the BellSteinberger relation, Phys. Rev. D 91, 015004 (2015).

[9] H. B. Li, Prospects for rare and forbidden hyperon decays at BESIII, Front. Phys. (Beijing) 12, 121301 (2017); Erratum 14, 64001 (2019).

[10] X. H. Hu and Z. X. Zhao, Study of the $s \rightarrow d \nu \bar{\nu}$ rare hyperon decays in the Standard Model and new physics, Chin. Phys. C 43, 093104 (2019).

[11] J. Tandean, Rare hyperon decays with missing energy, J. High Energy Phys. 04 (2019) 104.

[12] C. Bird, P. Jackson, R. V. Kowalewski, and M. Pospelov, Search for Dark Matter in $b \rightarrow s$ Transitions with Missing Energy, Phys. Rev. Lett. 93, 201803 (2004).

[13] C. Bird, R. V. Kowalewski, and M. Pospelov, Dark matter pair-production in $b \rightarrow s$ transitions, Mod. Phys. Lett. A 21, 457 (2006).

[14] X. G. He, S. Y. Ho, J. Tandean, and H. C. Tsai, Scalar dark matter and standard model with four generations, Phys. Rev. D 82, 035016 (2010).

[15] S. N. Gninenko and N. V. Krasnikov, Invisible $K_{L}$ decays as a probe of new physics, Phys. Rev. D 92, 034009 (2015).
[16] A. Badin and A. A. Petrov, Searching for light Dark Matter in heavy meson decays, Phys. Rev. D 82, 034005 (2010).

[17] J. F. Kamenik and C. Smith, FCNC portals to the dark sector, J. High Energy Phys. 03 (2012) 090.

[18] M. Arteaga, E. Bertuzzo, C. Caniu Barros, and Z. Tabrizi, Operators from flavored dark sectors running to low energy, Phys. Rev. D 99, 035022 (2019).

[19] X. G. He, J. Tandean, and G. Valencia, Implications of a new particle from the HyperCP data on $\Sigma^{+} \rightarrow p \mu^{+} \mu^{-}$, Phys. Lett. B 631, 100 (2005).

[20] M. Bourquin et al. (Bristol-Geneva-Heidelberg-OrsayRutherford-Strasbourg Collaboration), Measurements of hyperon semileptonic decays at the CERN Super Proton Synchrotron. 1. The $\Sigma^{-} \rightarrow \Lambda e^{-} \bar{\nu}$ decay mode, Z. Phys. C 12, 307 (1982).

[21] J. M. Gaillard and G. Sauvage, Hyperon beta decays, Annu. Rev. Nucl. Part. Sci. 34, 351 (1984).

[22] S. Y. Hsueh et al., A high precision measurement of polarized sigma- beta decay, Phys. Rev. D 38, 2056 (1988).

[23] J. Dworkin et al., High statistics measurement of $g_{a} / g_{v}$ in $\Lambda \rightarrow p+e^{-}+\bar{\nu}$, Phys. Rev. D 41, 780 (1990).

[24] J. R. Batley et al. (NA48/I Collaboration), Measurement of the branching ratios of the decays $\Xi^{0} \rightarrow \Sigma^{+} e^{-} \bar{\nu}_{e}$ and $\bar{\Xi}^{0} \rightarrow \Sigma^{-} e^{+} \nu_{e}$, Phys. Lett. B 645, 36 (2007).

[25] J. Charles, O. Deschamps, S. Descotes-Genon, H. Lacker, A. Menzel, S. Monteil, V. Niess, and J. Ocariz et al., Current status of the Standard Model CKM fit and constraints on $\Delta F=2$ new physics, Phys. Rev. D 91, 073007 (2015). Online updates available at http://ckmfitter.in2p3.fr.

[26] C. Bobeth and A. J. Buras, Leptoquarks meet $\epsilon^{\prime} / \epsilon$ and rare Kaon processes, J. High Energy Phys. 02 (2018) 101.

[27] B. Bhattacharya, C. M. Grant, and A. A. Petrov, Invisible widths of heavy mesons, Phys. Rev. D 99, 093010 (2019).

[28] L.S. Littenberg and G. Valencia, The decays $K \rightarrow \pi \pi \nu \bar{\nu}$ within the standard model, Phys. Lett. B 385, 379 (1996).

[29] C. W. Chiang and F. J. Gilman, $K_{L, S} \rightarrow \pi \pi \nu \bar{\nu}$ decays within and beyond the standard model, Phys. Rev. D 62, 094026 (2000).

[30] H. K. Dreiner, H. E. Haber, and S. P. Martin, Two-component spinor techniques and Feynman rules for quantum field theory and supersymmetry, Phys. Rep. 494, 1 (2010). 